\title{
QSO winds and galaxy evolution
}

\author{
M.J. Page*, F.J. Carrera ${ }^{\dagger}$ and J.A. Stevens ${ }^{* *}$ \\ *UCL, Mullard Space Science Laboratory, Holmbury St. Mary, Dorking, Surrey RH5 6NT, UK \\ ${ }^{\dagger}$ Instituto de Física de Cantabria, Avenida de los Castros, 39005 Santander, Spain \\ ${ }^{* *}$ Centre for Astrophysics Research, University of Hertfordshire, College Lane, Hatfield AL10 9AB, \\ $U K$
}

\begin{abstract}
There exists a population of broad-line $\mathrm{z} \sim 2$ QSOs which have heavily absorbed X-ray spectra. These objects constitute $\sim 10 \%$ of the population at luminosities and redshifts characteristic of the main producers of QSO luminosity in the Universe. Our follow up observations in the submm show that these QSOs are often embedded in ultraluminous starburst galaxies, unlike most unabsorbed QSOs at the same redshifts and luminosities. The radically different star formation properties between the absorbed and unabsorbed QSOs implies that the X-ray absorption is unrelated to the torus invoked in AGN unification schemes. Instead, these results suggest that the objects represent a transitional phase in an evolutionary sequence relating massive black holes and the formation of galaxies. The most puzzling question about these objects has always been the nature of the X-ray absorber. We present our study of the X-ray absorbers based on deep (50-100ks) XMM-Newton spectroscopy. We show that the absorption is most likely due to a dense ionised wind driven by the QSO. This wind could be the mechanism by which the QSO terminates the star formation in the host galaxy, and ends the supply of accretion material, to produce the present day black hole/spheroid mass ratio.
\end{abstract}

Keywords: X-rays: galaxies - galaxies: active - galaxies: evolution - galaxies: formation PACS: 98.54.-h, 98.54.Aj, 98.54.Ep

\section{INTRODUCTION}

The prevalence of black holes in present day galaxy bulges, and the proportionality between black hole and spheroid mass [8] implies that the formation of the two components are intimately linked. One way to probe star formation in distant QSOs is to observe them at submillimetre wavelengths, and so measure the amount of radiation from young stars which is absorbed and re-emitted by dust in the far infrared. With this in mind, we observed matched samples of X-ray absorbed and unabsorbed QSOs at $850 \mu \mathrm{m}$ with SCUBA. These observations revealed a remarkable dichotomy in the submillimetre properties of these two groups of sources: almost all of the X-ray absorbed QSOs at $z>1.5$ are ultraluminous infrared galaxies, while the X-ray unabsorbed QSOs are not $[10,13]$. This suggests that the two types are linked by an evolutionary sequence, whereby the QSO emerges at the end of the main star-forming phase of a massive galaxy $[13,16,5]$.

However, the nature of the X-ray absorption remains puzzling. These objects are characterised by hard, absorbed X-ray spectra, and assuming that this is due to photoelectric absorption from cold material with solar abundances, their column densities are $\sim 10^{22} \mathrm{~cm}^{-2}$. On the other hand, they have optical/UV spectra which are typical for QSOs, with broad emission lines and blue continua. For a Galactic gas/dust ratio, the 
restframe ultraviolet spectra would be heavily attenuated by such large columns of material, so the absorbers appear to contain very little dust. The X-ray absorption could be due to gas located within the AGN structure, or from more distant material in the host galaxy, but in either case the lack of dust is suprising. If the absorption were associated with the obscuring dusty torus invoked in unification schemes [2] we would expect significant dust attenuation, while the detection of the dust continuum at long wavelengths implies that the interstellar media of the host galaxies are also dust-rich. In order to investigate the X-ray absorption, we have therefore obtained deep (50-100ks) $X M M-N e w t o n$ observations of five submillimetre bright, X-ray absorbed QSOs from our sample of hard-spectrum Rosat sources [12].

\section{RESULTS}

For each QSO, the EPIC MOS and pn X-ray spectra were combined as described in Page, Davis \& Salvi [11]. The resulting spectra contain between 200 and 1100 counts. As a starting point, we fitted the X-ray spectra with models consisting of a power law and fixed Galactic absorption. Surprisingly, in most cases this model produces acceptable values of $\chi^{2} / v$. However, the best fit photon indices are unusually hard for QSOs: three of the five objects have $\Gamma \sim 1.4$ with uncertainties of $<0.1$ (see Fig. 1), while the other two have $\Gamma$ of 1.6 and 1.8, compared to the $\Gamma=1.9$ that is typical for X-ray selected QSOs [e.g. 7, 14]. Furthermore, the data show a deficit of counts relative to the model at the softest energies, indicating that absorption is present. The original Rosat PSPC spectra and the XMM-Newton spectra show excellent agreement (see Fig. 1). The 2-10 $\mathrm{keV}$ luminosities of the QSOs range from $3 \times 10^{44} \mathrm{ergs} \mathrm{s}^{-1}$ to $2 \times 10^{45} \mathrm{ergs} \mathrm{s}^{-1}$.

The hypothesis of a normal $(\Gamma=1.9)$ AGN X-ray spectrum and a cold absorber is rejected for the sample as a whole. Therefore we considered ionised absorber models for the X-ray absorption, using the 'xabs' model in SPEX, which includes both photoelectric and line absorption. For all five AGN, acceptable fits can be obtained with a $\Gamma=1.9$ power law and an absorber with an ionisation parameter log $\xi$ between 2 and 3, and column densities of $10^{22.5}-10^{23.5} \mathrm{~cm}^{-2}$. These absorbers have similar properties to the high-ionisation absorber phases seen as outflows in some nearby Seyfert 1 galaxies and QSOs such as NGC 3783 [9], H 0557-385 [3] and PG 1114+445 [4].

At these ionisation parameters and column densities, the absorbers are likely to originate in the AGN themselves, rather than in the host galaxies. This solution is attractive, because it is compatible with the lack of optical extinction in these objects: if the absorber is driven as a wind, either from the accretion disc or from evaporation of the inner edge of the molecular torus, then dust will be sublimated before (or as) it enters the flow.

With the hypothesis of an outflowing, ionised wind in mind, we now turn to the optical spectra of our five QSOs, which correspond to the UV in the restframe of the QSOs. It is notable that all five objects show significant absorption lines of C IV (e.g. Fig. 2), in most cases blue-shifted with respect to the systemic redshifts determined from the emission lines. Noting that the ionised X-ray absorbers in nearby Seyferts are normally accompanied by absorption lines in the UV, and assuming that the UV absorption lines in our QSOs are associated with the X-ray absorbers, we can use the UV absorption line 

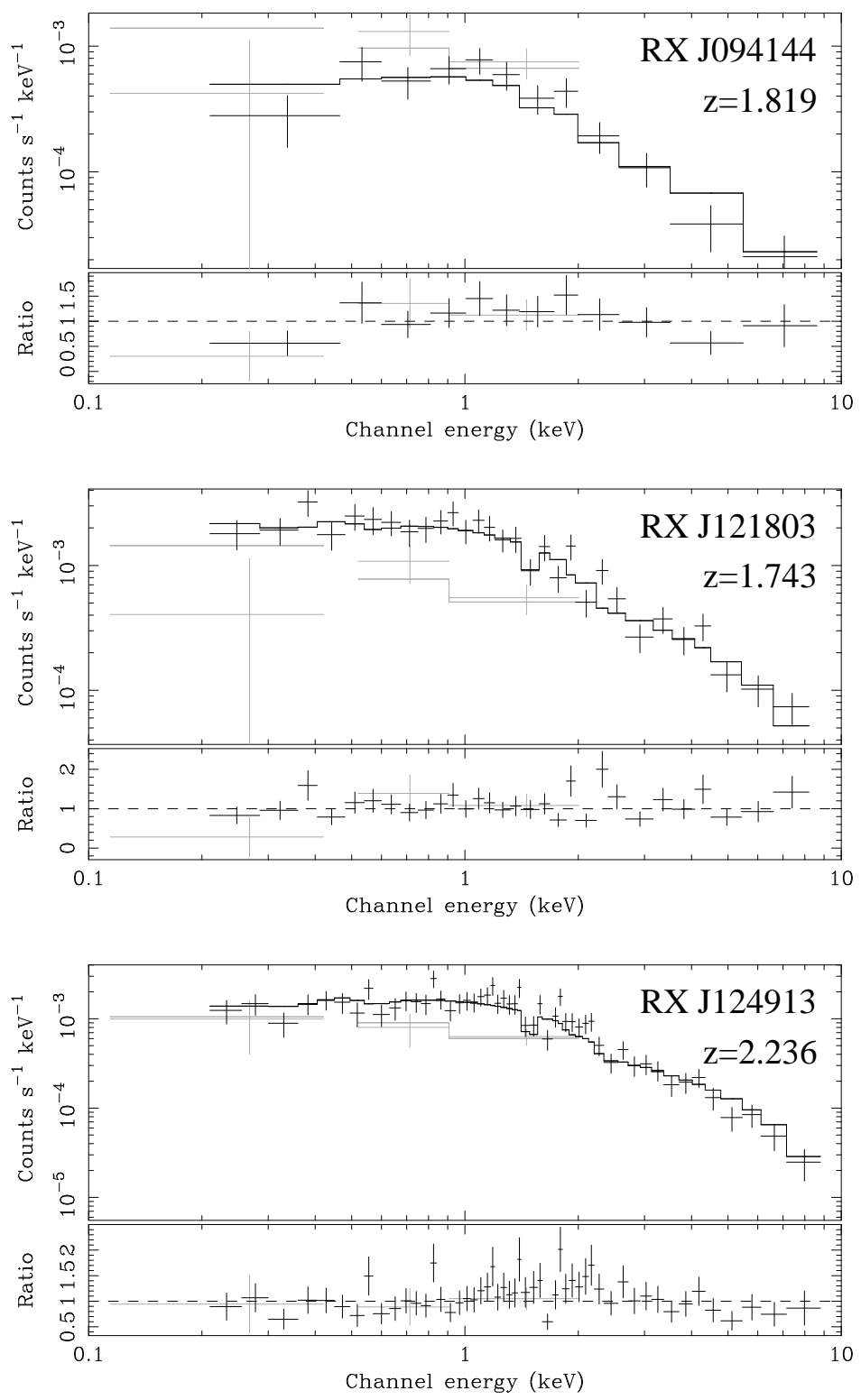

FIGURE 1. XMM-Newton EPIC spectra (black) and Rosat PSPC spectra (grey) of three X-ray absorbed QSOs. The model is a simple power law with fixed Galactic absorption. Compared to normal QSOs, the spectra are deficient in counts at low energy, indicating that absorption is responsible for the hard spectral shape; this is also seen in the Rosat data. Furthermore, RX J094144 and RX J124913 show some systematic curvature relative to the power law model.

velocities to investigate the likely kinematics and energetics of the X-ray absorbers. We also assume that the absorbers have similar filling factors $(\sim 0.01)$ to ionised absorbers with comparable ionisation parameters in well studied, nearby AGN [3]. We find that for a typical X-ray absorbed QSO, with a wind outflow velocity of $8000 \mathrm{~km} \mathrm{~s}^{-1}$, the mass outflow rate in the wind is about 10 times the accretion rate, and the kinetic power of the wind is equivalent to $\sim 4 \%$ of the radiative luminosity. 

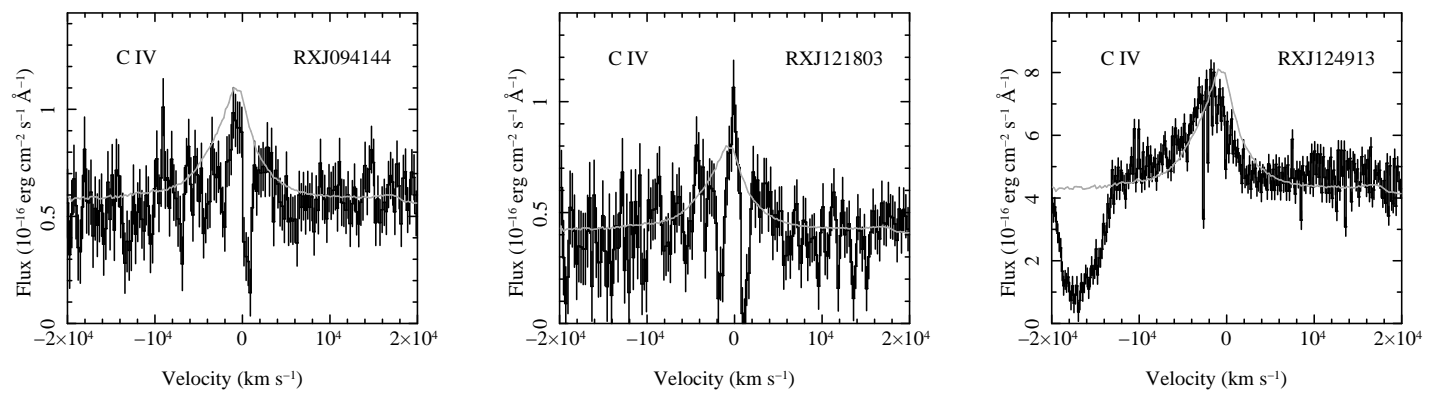

FIGURE 2. The rest-frame ultraviolet spectra of RX J094144, RX J121803 and RX J124913 around the C IV emission line, exhibiting strong absorption lines of C IV. The grey line shows the median QSO template from Vanden Berk et al. [17].

\section{IMPLICATIONS FOR AGN AND GALAXY EVOLUTION}

The low space density of X-ray absorbed QSOs relative to unabsorbed QSOs and to distant ultraluminous galaxies detected in blank field SCUBA surveys, implies that the $\mathrm{X}$-ray absorbed QSOs are caught during a short-lived transitional phase. Before this brief phase, AGN must be weak, and heavily obscured [1]; after this phase the host galaxy is essentially fully formed, and the naked QSO shines brightly until its fuel is consumed. A number of theoretical models predict a very similar evolutionary pattern. In many of these models, the QSO terminates the star formation in the host galaxy by driving a powerful wind [e.g. 15,6]. The EPIC spectra of our X-ray absorbed QSOs suggest that the absorbers are ionised winds driven by the AGN, and therefore that the transition between buried AGN and naked QSO could be mediated by a radiatively driven wind from the AGN, as predicted by these models.

\section{REFERENCES}

1. Alexander D.M., et al., 2005, Nature, 433, 604

2. Antonucci R., 1993, Annu.Rev. A\&A, 31, 473

3. Ashton C.E., Page M.J., Branduardi-Raymont G., Blustin A.J., 2006, MNRAS, 366, 521

4. Ashton C.E., et al., 2004, MNRAS, 355, 73

5. Carrera F.J., et al., 2006, ESA SP-604, 509

6. Di Matteo T., Springel V. \& Hernquist L., 2005, Nature, 433, 604

7. Mateos S., et al., 2005, A\&A, 433, 855

8. Merritt D. \& Ferrarese L., 2001, MNRAS, 320, L30

9. Netzer H., et al., 2003, ApJ, 599, 933

10. Page M.J., Stevens J.A.S., Mittaz J.P.D., Carrera F.J., 2001, Science, 294, 2516

11. Page M.J., Davis S.W. \& Salvi N.J., 2003, MNRAS, 343, 1241

12. Page M.J., Mittaz J.P.D. \& Carrera F.J., 2001, MNRAS, 325, 575

13. Page M.J., Stevens J.A., Ivison R.J., Carrera F.J., 2004, ApJ, 611, L85

14. Page M.J., et al. 2006, MNRAS, 369, 156

15. Silk J. \& Rees M., 1998, A\&A, 331, L1

16. Stevens J.A., et al., 2005, MNRAS, 360, 610

17. Vanden Berk D.E., et al., 2001, AJ, 122, 549 